\title{
Breast Conservative Therapy for Multifocal-Multicentric Breast Cancers
}

\author{
Chaitra Sonthineni $^{1} \cdot$ A. Verma ${ }^{1} \cdot$ Gaurav Agarwal $^{1}$
}

Published online: 5 May 2016

(c) Société Internationale de Chirurgie 2016

We read with interest the study concerning breast conservation surgery/treatment (BCT) for multifocal and multicentric breast cancer (MFMCBC) by Tan et al. [1]. It is a commendable effort to undertake BCT in MFMCBC, as not many centres can boast of $85 \%$ BCT rates in MFMCBC. There are a few comments and queries in our mind regarding this study, and we would appreciate further details and clarifications.

The study clubs multifocal and multicentric breast carcinomas together as a single group as opposed to some other studies [2,3], which were also quoted by the authors in their references, that tend to separate the two entities. It would have added more to our knowledge if the authors would have mentioned the details of these groups separately. What was the rationale of clubbing these relatively different groups, when other studies have reported very different rates of BCT in these two groups?

It would be interesting to know what impact breast segmentation had on the study as opposed to taking the more conventional breast quadrants in classifying MFMCBC, especially because the two groups, multifocal tumours and multicentric tumours, had been taken as one single entity, thereby making any differentiation between multifocal and multicentric tumours unnecessary. What was the rate of BCT in multicentric tumour cohort, as opposed to the multifocal cohort in their experience? Such information will allow a more robust comparison with other published studies, most of which define multicentric disease on the basis of quadrants. In the event both the groups had equally high rates of

Chaitra Sonthineni

chaitra_chaitu@yahoo.co.in

1 Department of Endocrine and Breast Surgery, Sanjay Gandhi Post Graduate Institute of Medical Sciences, Lucknow, India
BCT in this study, could the high BCT rate in multicentric tumours be on account of the fact that the authors have defined multicentricity based on segments and not quadrants? It is possible that only a few of their patients may have had multiple tumours in different quadrants (which is the more classical definition of multicentricity) - which would make BCT even more difficult compared to multiple tumours in different segments (which may or may not be a part of the same quadrant). Nothing, can be said of this as further details were not provided by the authors, leaving a lot to speculation.

One of the most appealing aspects of this study was the categorising of mastectomy patients as 'mastectomy by choice' and 'mastectomy by need'. This truly reflects the necessity of developing more bold ways, such as the author's, of attaining high BCT rates in patients who wish for it.

We are curious to know how many patients in the study had the intervening tissue between the two breast lumps positive for malignancy. Such details might shed some light on the true nature of multicentric tumours. We hope the authors continue their endeavour and add further wisdom to the existing management options of MFMCBC.

\section{References}

1. Tan MP, Sitoh NY, Sitoh YY (2016) Optimising breast conservation treatment for multifocal and multicentric breast cancer: a worthwhile endeavour? World J Surg 40:315-322

2. Ataseven Beyhan et al (2015) Impact of multifocal or multicentric disease on surgery and locoregional, distant and overall survival of 6,134 breast cancer patients treated with neoadjuvant chemotherapy. Ann Surg Oncol 22:1118-1127

3. Wolters et al (2013) Comparing the outcome between multicentric and multifocal breast cancer: what is the impact on survival, and is there a role for guideline-adherent adjuvant therapy? A retrospective multicenter cohort study of 8,935 patients. Breast Cancer Res Treat 142:579-590 